Baran Marzena, Miziak Paulina, Bonio Katarzyna. Characteristic of warfarin. Journal of Education, Health and Sport. 2020;10(8):78-80. eISSN 2391-8306. DOI http://dx.doi.org/10.12775/JEHS.2020.10.08.009

https://apcz.umk.pl/czasopisma/index.php/JEHS/article/view/JEHS.2020.10.08.009

https://zenodo.org/record/3969341

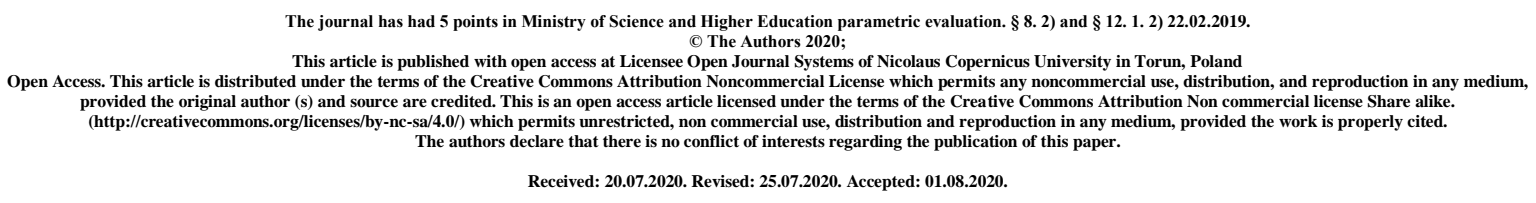

\title{
Characteristic of warfarin
}

\author{
Marzena Baran', Paulina Miziak ${ }^{1}$, Katarzyna Bonio ${ }^{2}$ \\ ${ }^{1}$ Chair and Department of Biochemistry and Molecular Biology, Medical University of Lublin \\ ${ }^{2}$ Department of Cell Biology, Institute of Biological Sciences, Maria Curie-Skłodowska University, Lublin
}

\begin{abstract}
In modern pharmacology, compounds of natural origin are gaining more and more recognition. One of them is warfarin, which is a compound of organic coumarin derivatives. It is used medicinally in the anticoagulant doctor. This compound was commercially introduced to the United States in the 1950s. It has been available on the Polish market since 2006. Warfarin is an antagonist of vitamin $\mathrm{K}$. It works by inhibiting the synthesis of vitamin $\mathrm{K}$ in the liver and thus blocking the synthesis of vitamin $\mathrm{K}$ dependent blood clotting factors (II, VII, IX, X). It is widely used by people suffering from cardiovascular diseases such as venous thrombosis and venous embolism. Adopted by patients with suspected or after a heart attack. Despite its undisputed positive effect, one should remember about the numerous side effects resulting from long-term use of warfarin. They may be due to intolerance to this compound, which causes nausea, vomiting, and allergic reactions. Moreover, recent studies show that it may have a destructive effect on the liver and cause calcification of blood vessels. Keep in mind that warfarin can interact with many substances you are taking for other medical conditions. Agents that increase the effects of warfarin include antibiotics such as erythromycin, deoxicillin, commonly used drugs containing caffeine, acetylsalicylic acid and naturally derived compounds found in ginkgo, garlic and sage. Compounds that weaken the effect of warfarin are barbiturates, cyclosporine, vitamin C, and St. John's wort.
\end{abstract}

Key words: warfarin, cumarine, vitamin $\mathrm{K}$ antagonist, medicine, anticoagulants 


\section{Characteristic and mechanism of action}

Warfarin is one of the most used anti-agglutinants. This compound belongs to the coumarins and is a vitamin $\mathrm{K}$ antagonist [1]. Anti-agglutination therapy is used in the treatment of blood clots, blockages in the pulses, heart attacks and atrial fibrillation [2]. The anticoagulant effect of warfarin is due to its chemical similarity to vitamin $\mathrm{K}$ [3]. It is a compound of natural origin derived from dicoumarol, which was first discovered in Galium odoratum [4]. It occurs in plants such as Melilotus officinalis from the Fabaceae family [4].

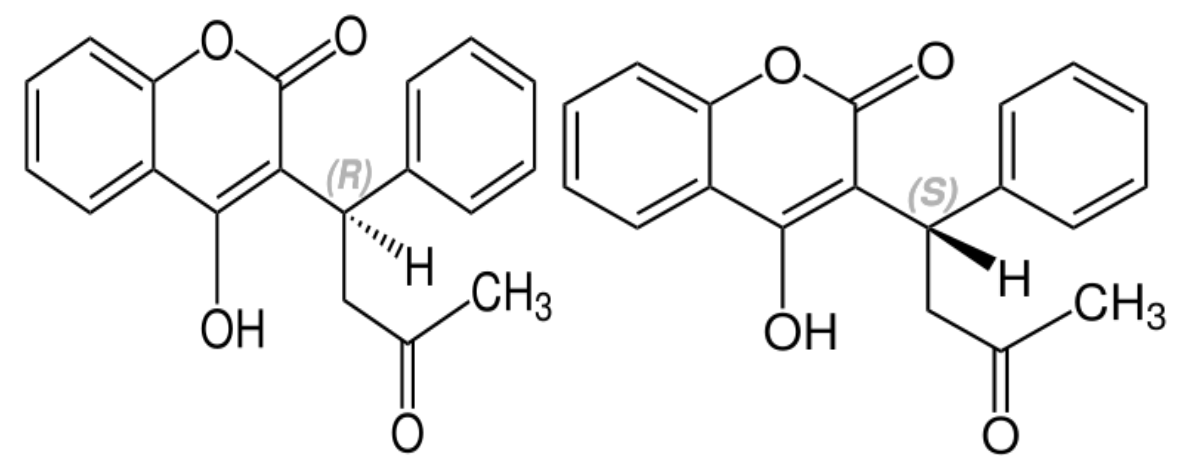

Figure 1. Structural formula of $R$ and $S$ warfarin.

Chemically warfarin consists of a racemic mixture of two R and S enantiomers (Fig.1) [2]. Both forms exist in equal proportions, but differ in potency, half-life, and metabolic pathway. The S-warfarin enantiomer has stronger effects [3]. This compound is quickly absorbed into the gastrointestinal tract, has a high bioavailability, and after 90 minutes it is found in the circulatory system [2]. In blood, warfarin binds to albumin [3]. Maintaining proper homeostasis of the body is a very complex process. Processes such as coagulation, anticoagulation and fibrinolysis take part in maintaining the balance [4]. Vitamin $\mathrm{K}$ is involved in the blood clotting process, which is essential for the synthesis of blood coagulation factors in the liver. These factors include prothrombin (factor II), factor VII, IX and $\mathrm{X}$ [3]. Warfarin, as a vitamin $\mathrm{K}$ antagonist, inhibits the action of reductase, which is responsible for the synthesis of hydroquinones from epoxide. Warfarin strongly affects especially epoxy reductases [5]. This disrupts the carboxylation of regulatory proteins that have an anti-agglutant effect. Warfarin competes with vitamin $\mathrm{K}$ for an active site in the hapatocyte. Therefore, in the absence of vitamin $\mathrm{K}$, inactive blood coagulation factors are formed [3]. The effect of this compound depends on the time of disintegration of individual blood coagulation factors [5]. As a rule, it is necessary to wait a few days for a satisfactory therapeutic effect [3]. The effectiveness of warfarin as a substance preventing the blood clotting process is dictated by several factors, such as the patient's age, the presence of comorbidities, the use of other drugs. The derivative of this coumarin may interact with other compounds of natural origin, increasing the risk of side effects. Herbs that interact particularly with warfarin include garlic, ginkgo, ginger, and St. John's wort. Other coumarins, vitamin K, terpenes and lignans have a key influence on treatment with warfarin. Summarizing all substances that can affect the agglutination process [2]. The compounds that weaken the effect of warfarin are mainly corticosteroids and non-steroidal anti-inflammatory drugs, which increase the synthesis of blood coagulation factors [2]. 


\section{Warfarin in medicine}

Anticoagulants are vitamin $\mathrm{K}$ antagonists. In modern medicine, they are used in anticoagulant treatment in a group of cardiac patients. This applies to patients with: permanent and paroxysmal atrial fibrillation and those with a heart valve. Warfarin, due to its potency and sustained adequate level in blood serum, is a frequently used drug in these diseases. Warfarin has a high bioavailability, is quickly absorbed from the gastrointestinal tract, and the maximum concentration in the blood is reached after 90 minutes. It binds to albumin in the blood, accumulates in the liver, and each isomer is metabolized through a different metabolic pathway [4]. Currently, warfarin is used to treat venous thrombosis and pulmonary embolism, and it can also prevent a heart attack. Additionally, it is used in long-term treatment to prevent thromboembolic events. However, bleeding may be a side effect of using this substance. Warfarin is also used in the treatment of osteoarthritis. However, it can be dangerous due to its narrow therapeutic index, interactions with other drugs and food, and because its users often have special clinical conditions [3]. One of the fundamental units in cardiology where anticoagulants are fully used is atrial fibrillation (AF)[4]. Further recommendations for warfarin therapy include patients with valvular disease [6]. This mainly applies to diseases of the mitral valve. In mitral regurgitation, treatment with anticoagulants should be used in patients with mitral valve insufficiency accompanied by paroxysmal or permanent AF, thromboembolic problems, in the presence of a left atrial thrombus and after mitral valve repair [4]. The increase in vascular calcification is caused by the inhibition of the enzyme matrix gamma-carboxyglutamate protein Gla (MGP). MGP is a vitamin K dependent protein that usually prevents systemic calcification by scavenging calcium phosphate in the tissues. Warfarin increases systemic calcification by acting on MGP. This calcification can cause the arteries and trachea and bronchi to calcify. Moreover, the use of warfarin has been associated with bone health disorders [1].

\section{References}

1. Poterucha T.J. i Goldhaber S.Z., Warfarin and Vascular Calcification, American Journal of Medicine, 2016, Vol 129, Issue 6

2. Nataša Milić i inni, Warfarin Interactions with Medicinal Herbs, Natural Product Communications, 2014, vol. 9, no. 8

3. Leite P.M. i inni, Review on mechanisms and interactions in concomitant use of herbs and warfarin therapy, Biomedicine and Pharmacotherapy, 2016, vol. 83

4. Sidłowska E. i Kozłowski D., Clinical use of warfarin in the cardiology, Geriatria, 2008, 2

5. Tales J.S. I inni, Warfarin: pharmacological profile and drug interactions with antidepressants Einstein (São Paulo), 2012, vol.10 no.1

6. European Journal of Cardiovascular Prevention and Rehabilitation 2007; 14(suppl 2): 8687. 